\title{
Article \\ Approaches to Three-Way Decisions Based on the Evaluation of Probabilistic Linguistic Terms Sets
}

\author{
Pingping Gu ${ }^{1} \mathbb{D}$, Jiubing Liu ${ }^{2}$ and Xianzhong Zhou ${ }^{1,3, *}$ \\ 1 School of Management and Engineering, Nanjing University, Nanjing 210023, China ; \\ gpingping@smail.nju.edu.cn \\ 2 School of Business, Shantou University, Shantou 515063, China; jiubingliu@163.com or jiubingliu@stu.edu.cn \\ 3 Research Center for Novel Technology of Intelligent Equipment, Nanjing University, Nanjing 210023, China \\ * Correspondence: zhouxz@nju.edu.cn
}

check for updates

Citation: Gu, P.; Liu, J.; Zhou, X. Approaches to Three-Way Decisions Based on the Evaluation of Probabilistic Linguistic Terms Sets. Symmetry 2021, 13, 764. https:// doi.org/10.3390/sym13050764

Academic Editor: José Carlos R. Alcantud

Received: 26 March 2021

Accepted: 24 April 2021

Published: 28 April 2021

Publisher's Note: MDPI stays neutral with regard to jurisdictional claims in published maps and institutional affiliations.

Copyright: (c) 2021 by the authors. Licensee MDPI, Basel, Switzerland. This article is an open access article distributed under the terms and conditions of the Creative Commons Attribution (CC BY) license (https:// creativecommons.org/licenses/by/ $4.0 /)$.

\begin{abstract}
The method of determining probability thresholds of three-way decisions (3WDs) has always been the key of research, especially in the current environment with a large number of data and uncertainties. Among these problems, there will be correlation and similarity between them. In the light of these problems, the loss function with Probabilistic Linguistic Terms Sets (PLTSs) is introduced in the paper, and then we propose a PLTS evaluation-based approach to determine the thresholds and derive 3WDs. According to the definition and characters of PLTSs, the PLTSs loss function matrix is constructed firstly. Then using the equivalent model of Decision-theoretic rough sets (DTRSs), we construct the equivalent model (i.e., the $\alpha^{\text {opt }}$-model and the $\beta^{\text {opt }}$-model, which are symmtrical) and try to find the optimal solution to determine the thresholds. Based on that, we propose a novel three-way decision approach under PLTSs evaluations. Finally, the validity of the method is verified by an example.
\end{abstract}

Keywords: three-way decisions; probability thresholds; probabilistic linguistic terms sets; equivalent model

\section{Introduction}

Three-way decisions, which were first proposed by Yiyu Yao in the early 1990s, is a new decision analysis method of dealing with inaccurate and incomplete information [1]. As an important promotion of traditional two-way decisions, it provides an effective strategy, which trisects decision problems into three decision semantics with positive domain (accept decision), negative domain (reject decision), and boundary domain (delayed decision) to solve complex decision problems. Based on the rough set theory model, Yao introduced the Bayes risk decision method to analyze and evaluate the risk cost of various decisions and then obtained the minimum risk cost evaluation decision result among them [2]. Nowadays, theories and methods related to three-way decisions have attracted widespread attention from scholars [3-7]. Moreover, it has been successfully applied to many disciplines and fields, such as attribute reduction [8-10], paper review [11], recommendation system [12,13], granular computing [3,14], multi-attribute decision-making [15], fuzzy clustering [16,17], concept learning [18,19], medical diagnosis [20] and face recognition [21].

In three-way decisions, determining the threshold pair $\alpha$ and $\beta$ is an important problem. Up to now, fruitful researches have been achieved under the thorough study of many scholars [2,22-24]. Yao constructed a decision rough set model based on Bayesian theory and then derived an analytical solution of probability threshold directly, which provided a reasonable semantic explanation for the threshold pair of Probabilistic Rough Sets model [2] Based on this, Li et al. deduced the expression of three decision thresholds-optimistic tendency, pessimistic tendency and neutral tendency-by introducing the risk preference of decision-makers [22]. Based on the research on the relationship between loss risk and threshold parameters in the basic model, Jia et al. proposed an optimization model and designed an adaptive learning algorithm to solve the model to determine the probability 
threshold [25]. In addition, based on the Game-Theoretic Rough Set model, a method of determining three decision probability thresholds was proposed by Azam et al [26]. Obviously, the above methods are mainly used to study the loss function with real value. Due to the uncertainty, complexity, and fuzziness of the real environment in practical application, decision-makers (DMs) or experts often give fuzzy or uncertain evaluation forms rather than specific real value evaluation in the loss function $[27,28]$, such as interval number (INs), triangle fuzzy number (TFs), Intuitionistic Fuzzy Sets (IFSs), and Pythagorean Fuzzy Sets (PFSs). Therefore, many scholars begin to extend the classical decision-theoretic rough sets (DTRSs) to the fuzzy or uncertain environment; they have put forward many extended decision rough set models in recent years.

Note that for multi-attribute decision-making and evaluation problems, such as project evaluation, personnel assessment, medical diagnosis, the current solving trend is still the logical inference based on prior knowledge. However, due to the complexity of objective things and the limitations of personal knowledge, it is impossible to describe objective things and their attribute characteristics accurately. In this case, fuzzy linguistic evaluation has gradually become a new choice for DMs. According to the flourishing development of fuzzy set theory, a large number of scholars have proposed a series of concepts: Linguistic Term Sets (LTSs) [29], Hesitant Fuzzy Sets(HFSs) [30], Hesitant Fuzzy Linguistic Term Sets (HFLTSs) [31], Probabilistic Linguistic Term Sets (PLTSs) [32], and Interval-valued Probabilistic Linguistic Term Sets (IVPLTSs) [33]. By describing the evaluation preference of decision-makers and measuring the degree of preference comprehensively, PLTSs (Qi, 2016) makes the decision result more reliable and practical [32]. Some progress on the application of PLTSs to decision-making has been recently; however, it is still in its infancy. Therefore, under the evaluation of PLTSs, it is also an important research problem that how to determine the threshold pair $\alpha$ and $\beta$.

This paper proposed a novel three-way decision approach under PLTSs evaluations to determine the threshold pair. The reminder of this paper is organized as follows. Section 2 briefly reviews the concepts of PLTSs, DTRSs, and the equivalent model of DTRSs. In Section 3, we describe the three-way decision problems based on evaluation of Probabilistic linguistic Term Sets. Based on the evaluation of PLTSs, a method for determining the threshold pair and rules of $3 \mathrm{WDs}$ is proposed in Section 4 . Section 5 presents an example to verify the validity of the proposed method. The conclusion of this paper is summarized in Section 6.

\section{Preliminaries}

In this section, we introduce some basic concepts and notations, such as PLTSs and their properties as well as DTRSs.

\subsection{Probabilistic Linguistic Term Sets}

Definition 1. Ref. [34] Let $S$ be a set with odd elements, a linguistic term set is defined as

$$
S=\left\{s_{\alpha}, \alpha=0,1, \cdots, \tau\right\},
$$

where $\tau$ is a positive integer, $s_{\alpha}$ is a linguistic term, and $s_{0}$ and $s_{\tau}$ represent the minimum and maximum of linguistic evaluation, respectively.

Based on Definition 1, we have

(1) Ordering: if $\alpha>\beta$, then $s_{\alpha}>s_{\beta}$.

(2) Complementarity: if $\alpha+\beta=\tau$, then $n e g\left(s_{\alpha}\right)=s_{\beta}$.

Definition 2. Ref. [34] Let $s_{\alpha}$ and $s_{\beta}$ be two linguistic term sets: $s_{\alpha}, s_{\beta} \in S$, parameter $\lambda, \lambda_{1}, \lambda_{2} \in$ $[0,1]$; then, we have the following operation rules:

$$
\text { (1) } s_{\alpha} \bigoplus s_{\beta}=s_{\beta} \bigoplus s_{\alpha}=s_{\alpha+\beta}
$$




$$
\begin{aligned}
(2)\left(\lambda_{1}+\lambda_{2}\right) s_{\alpha} & =\lambda_{1} s_{\alpha} \bigoplus \lambda_{2} s_{\alpha} \\
\text { (3) } \lambda\left(s_{\alpha} \bigoplus s_{\beta}\right) & =\lambda s_{\alpha} \bigoplus \lambda s_{\beta}
\end{aligned}
$$

$$
\text { (4) } \lambda s_{\alpha}=s_{\lambda \alpha}
$$

As a linguistic term set can only represent a range of evaluation levels rather than an accurate level, DMs seem to hesitate about choosing from multiple decisions. Therefore, the hesitant fuzzy linguistic term set was put forward by Rodriguez et al. [35].

Definition 3. Ref. [35] Let $S$ be a set of the evaluation objects, $C$ be an attribute set of the evaluation objects, and $S=\left\{s_{\alpha}, \alpha=0,1, \cdots, \tau\right\}$ be a LTSs, then a HFLTS $H(x \mid c)$ is an ordered finite subset of consecutive linguistic terms of $S$ with $\tau_{x}+1$ linguistic terms; it can be defined as follows:

$$
H(x \mid c)=\left\{s_{i_{x}}, s_{i_{x}+1}, \cdots, s_{i_{x}+\tau_{x}}\right\}
$$

Recently, Zhu and Xu defined some basic operations of HFLTSs as follows [36]:

Definition 4. Ref. [36] Given two HFLTSs: $H_{\alpha}\left\{h_{\alpha}^{l} \mid l=1,2, \cdots, \# H_{\alpha}\right\}$ and $H_{\beta}\left\{h_{\beta}^{l} \mid l=1,2\right.$, $\left.\cdots, \# H_{\beta}\right\}, \# H_{\alpha}=\# H_{\beta}, \lambda \geq 0$, then

$$
\begin{gathered}
(1) H_{\alpha} \bigoplus H_{\beta}=\cup_{h_{\alpha}^{l} \in H_{\alpha}, h_{\beta}^{l} \in H_{\beta}}\left\{h_{\alpha}^{l} \bigoplus h_{\beta}^{l}\right\} \\
\text { (2) } \lambda H_{\alpha}=\cup_{h_{\alpha}^{l} \in H_{\alpha}}\left\{\lambda h_{\alpha}^{l}\right\}
\end{gathered}
$$

where $h_{\alpha}^{l}$ and $h_{\beta}^{l}$ are the lth linguistic terms in $H_{\alpha}$ and $H_{\beta}$, respectively, and $\# H_{\alpha}$ and $\# H_{\beta}$ are the numbers of the linguistic terms in $H_{\alpha}$ and $H_{\beta}$, respectively.

Considering the uncertainty of linguistic terms in $H(x \mid c)$ and the preference of DMs, Pang et al. introduced probability into linguistic terms and then presented the following concept of PLTS:

Definition 5. Ref. [32] Let $S=\left\{s_{\alpha}, \alpha=0,1, \cdots, \tau\right\}$ be a LTS; a PLTS is defined as

$$
L(p)=\left\{L^{(k)}\left(p^{(k)}\right) \mid L^{(k)} \in S, p^{(k)} \geq 0, k=1,2, \cdots, \# L(p), \sum_{k=1}^{\# L(p)} p^{(k)} \leq 1\right\}
$$

where $L^{(k)}\left(p^{(k)}\right.$ is the linguistic term $L^{(k)}$ associated with the probability $p^{(k)}$ and $\# L(p)$ is the number of all different linguistic terms in $L(p)$.

In particular, when $\sum_{k=1}^{\# L(p)} p^{(k)}=1$, we have the complete information of probabilistic distribution of all possible linguistic terms, when $\sum_{k=1}^{\# L(p)} p^{(k)}<1$, partial ignorance exist owing to the limitation of current knowledge. Therefore, we need to normalize the PLTSs.

Definition 6. Ref. [32] Given a PLTS L $(p)$ with $\sum_{k=1}^{\# L(p)} p^{(k)}<1$, the associated PLTS L $(\dot{p})$ is defined by

$$
L(\dot{p})=\left\{L^{(k)}\left(\dot{p}^{(k)} \mid k=1,2, \cdots, \# L(p)\right\}\right.
$$

where $\dot{p}^{(k)}=\frac{p^{(k)}}{\sum_{k=1}^{\# L(p)} p^{(k)}}$.

Definition 7. Ref. [32] Let $L_{1}(p)$ and $L_{2}(p)$ be two PLTSs; $\# L_{1}(p)$ and $\# L_{2}(p)$ denote the numbers of the linguistic terms in $L_{1}(p)$ and $L_{2}(p)$, respectively. If $\# L_{1}(p)>\# L_{2}(p)$, in order to make the number of linguistic terms in $L_{1}(p)$ and $L_{2}(p)$ be identical, we will add $\# L_{1}(p)-\# L_{2}(p)$ 
linguistic terms to $L_{2}(p)$. The added linguistic terms are the smallest ones, and their probabilities are zero.

Given two PLTSs, $L_{1}(p)=\left\{L_{1}^{(k)}\left(p_{1}^{(k)}\right), k=1,2, \cdots, \# L_{1}(p)\right\}$ and $L_{2}(p)=\left\{L_{2}^{(k)}\left(p_{2}^{(k)}\right)\right.$, $\left.k=1,2, \cdots, \# L_{2}(p)\right\}$, the normalization process can be shown by the following steps:

(1) If $\sum_{k=1}^{\# L_{i}(p)} p_{i}^{(k)}<1$, then we calculate $L_{i}(\dot{p}), i=1,2$ by the Definition 6 .

(2) If $\# L_{1}(p) \neq \# L_{2}(p)$, then according to the Definition 7 , some elements are added to the one with smaller number of elements.

Simultaneously, Pang et al. defined some basic operations of PLTSs as follows [32]:

Definition 8. Ref. [32] Let $L_{1}(p)$ and $L_{2}(p)$ be two PLTSs, and $\# L_{1}(p)=\# L_{2}(p)$; then, we have the following operation rules:

$$
L_{1}(p) \bigoplus L_{2}(p)=\cup_{L_{1}^{(k)} \in L_{1}(p), L_{2}^{(k)} \in L_{2}(p)}\left\{p_{1}^{(k)} L_{1}^{(k)} \bigoplus p_{2}^{(k)} L_{2}^{(k)}\right\}
$$

The following is the other operational law in PLTSs:

$$
\lambda L(p)=\cup_{L^{(k)} \in L(p)} \lambda p^{(k)} L^{(k)}
$$

\subsection{Decision-Theoretic Rough Sets}

In Decision-theoretic rough sets, $S=\{C, \neg C\} \triangleq\{P, N\}$ is supposed to be two states that the object belongs to or belongs to $C$ or $\neg C[1,2,37]$. In addition, the set of actions is represented by $A=\left\{a_{P}, a_{B}, a_{N}\right\}$, where $a_{P}, a_{B}$, and $a_{N}$ indicate three decision actions for classifying an object, which correspond to $x \in P O S(C), x \in N E G(C)$, and $x \in B N D(C)$ in the decision-making process. The loss function in the actual decision-making process of taking different actions in two states is shown in Table 1 , where $\lambda_{P P}, \lambda_{B P}$, and $\lambda_{N P}$ denote the losses generated by taking actions $a_{P}, a_{B}$, and $a_{N}$, respectively.

Table 1. Decision cost loss function.

\begin{tabular}{ccc}
\hline & $C(P)$ & $\neg C(N)$ \\
\hline$a_{P}$ & $\lambda_{P P}=\lambda\left(a_{P} \mid C\right)$ & $\lambda_{P N}=\lambda\left(a_{P} \mid \neg C\right)$ \\
$a_{B}$ & $\lambda_{B P}=\lambda\left(a_{B} \mid C\right)$ & $\lambda_{B N}=\lambda\left(a_{B} \mid \neg C\right)$ \\
$a_{B}$ & $\lambda_{N P}=\lambda\left(a_{N} \mid C\right)$ & $\lambda_{N N}=\lambda\left(a_{N} \mid \neg C\right)$ \\
\hline
\end{tabular}

Meanwhile, $\operatorname{Pr}(C \mid[o])$ and $\operatorname{Pr}(\neg C \mid[o])$ represent the conditional probability of an object $o$ belonging to $C$ or $\neg C$, and $\operatorname{Pr}(C \mid[o])+\operatorname{Pr}(\neg C \mid[o])=1$. Therefore, the expected losses for each object $o$ are calculated as

$$
R\left(a_{\bullet} \mid[o]\right)=\lambda_{\bullet P} \operatorname{Pr}(C \mid[o])+\lambda_{\bullet N} \operatorname{Pr}(\neg C \mid[o])
$$

For the minimum decision cost, the following decision rules are obtained based on the Bayesian decision procedure:

(P) If $R\left(a_{P} \mid[o]\right) \leq R\left(a_{B} \mid[o]\right)$ and $R\left(a_{P} \mid[o]\right) \leq R\left(a_{N} \mid[o]\right)$, then $o \in \operatorname{POS}(C)$.

(B) If $R\left(a_{B} \mid[o]\right) \leq R\left(a_{P} \mid[o]\right)$ and $R\left(a_{B} \mid[o]\right) \leq R\left(a_{N} \mid[o]\right)$, then $o \in B N D(C)$.

(N) If $R\left(a_{N} \mid[o]\right) \leq R\left(a_{P} \mid[o]\right)$ and $R\left(a_{N} \mid[o]\right) \leq R\left(a_{B} \mid[o]\right)$, then $o \in N E G(C)$.

The above rules $(\mathrm{P})-(\mathrm{N})$ are called three-way decisions (3WDs), in other words accepted, rejected, and delayed decision [2,38]. Considering the practical applications, a reasonable case of the loss function is often given as $\lambda_{P P} \leq \lambda_{B P} \leq \lambda_{N P}$ and $\lambda_{N N} \leq \lambda_{B N} \leq \lambda_{P N}$.

Thus, the decision rules (P)-(N) can be further simplified as

(P1) If $\operatorname{Pr}(C \mid[o]) \geq \alpha$, then $o \in \operatorname{POS}(C)$.

(B1) If $\beta<\operatorname{Pr}(C \mid[o])<\alpha$, then $o \in B N D(C)$.

(N1) If $\operatorname{Pr}(C \mid[o]) \leq \beta$, then $o \in N E G(C)$. 
The thresholds are computed by

$$
\begin{array}{r}
\alpha=\frac{\lambda_{P N}-\lambda_{B N}}{\left(\lambda_{P N}-\lambda_{B N}\right)+\left(\lambda_{B P}-\lambda_{P P}\right)} \\
\beta=\frac{\lambda_{B N}-\lambda_{N N}}{\left(\lambda_{B N}-\lambda_{N N}\right)+\left(\lambda_{N P}-\lambda_{B P}\right)} \\
\gamma=\frac{\lambda_{P N}-\lambda_{N N}}{\left(\lambda_{P N}-\lambda_{N N}\right)+\left(\lambda_{N P}-\lambda_{P P}\right)}
\end{array}
$$

However, two cases may occur in accordance with the above calculation: (i) $\alpha>\beta$ and (ii) $\alpha \leq \beta$. Considering the former first, that is,

(i) $\alpha>\beta$, which means $\left(\lambda_{B P}-\lambda_{P P}\right)\left(\lambda_{B N}-\lambda_{N N}\right)<\left(\lambda_{P N}-\lambda_{B N}\right)\left(\lambda_{N P}-\lambda_{B P}\right)$. The rules (P1)-(N1) can be further simplified as

(P2) If $\operatorname{Pr}(C \mid[o]) \geq \alpha$, then $o \in \operatorname{POS}(C)$.

(B2) If $\beta<\operatorname{Pr}(C \mid[o])<\alpha$, then $o \in B N D(C)$.

(N2) If $\operatorname{Pr}(C \mid[o]) \leq \beta$, then $o \in N E G(C)$.

(ii) $\alpha \leq \beta$, which means $\left(\lambda_{B P}-\lambda_{P P}\right)\left(\lambda_{B N}-\lambda_{N N}\right) \geq\left(\lambda_{P N}-\lambda_{B N}\right)\left(\lambda_{N P}-\lambda_{B P}\right)$.

(P3) If $\operatorname{Pr}(C \mid[o]) \geq \gamma$, then $o \in \operatorname{POS}(C)$.

(N3) If $\operatorname{Pr}(C \mid[o])<\beta$, then $o \in N E G(C)$.

In order to explain the basic ideals, Figure 1 shows the trisecting-and-acting model in Decision-theoretic rough sets. The triplet (Region 1, Region 2, Region 3) is called a tri-partition of a universal set and a bisection of the set is a special case in it.
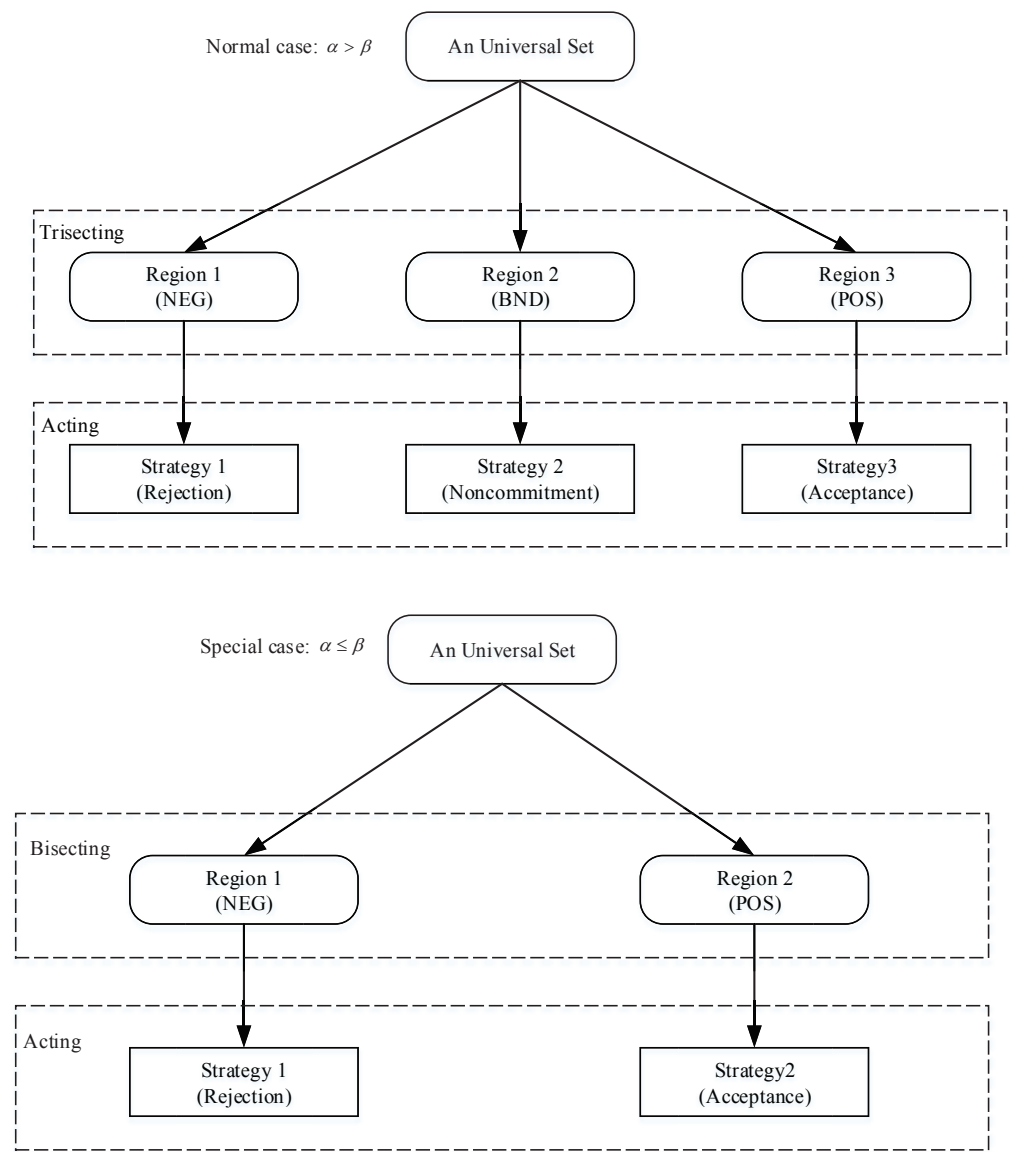

Figure 1. Trisecting-and-acting model. 


\subsection{Equivalent Model of Decision-Theoretic Rough Sets}

The conditional probabilities of an object belongs to $C$ or $\neg C$ are recorded as $g=$ $\operatorname{Pr}(C \mid[o])$ and $h=\operatorname{Pr}(\neg C \mid[o])$, respectively; $g+h=1$. Based on the DTRSs, Liu et al. proposed the following equivalent model [39]:

$$
\begin{aligned}
& \alpha^{(o p t)}-\text { model }: \\
& \alpha^{(o p t)}=\min g \\
& \text { s.t. }\left\{\begin{array}{l}
g \lambda_{P P}+h \lambda_{P N} \leq g \lambda_{B P}+h \lambda_{B N} \\
g \lambda_{P P}+h \lambda_{P N} \leq g \lambda_{N P}+h \lambda_{N N} \\
g+h=1, h \geq 0
\end{array}\right. \\
& \beta^{(o p t)}-\text { model : } \\
& \beta^{(o p t)}=\max g \\
& \text { s.t. }\left\{\begin{array}{l}
g \lambda_{N P}+h \lambda_{N N} \leq g \lambda_{P P}+h \lambda_{P N} \\
g \lambda_{N P}+h \lambda_{N N} \leq g \lambda_{B P}+h \lambda_{B N} \\
g+h=1, h \geq 0
\end{array}\right.
\end{aligned}
$$

In Equation (15), according to the rules (P) and (P2), the threshold $\alpha$ is the minimum $g$ of all conditional probabilities, which satisfies $R\left(a_{P} \mid[o]\right) \leq R\left(a_{B} \mid[o]\right)$ and $R\left(a_{P} \mid[o]\right) \leq$ $R\left(a_{N} \mid[o]\right)$. In Equation (16), according to the rules (N) and (N2), the threshold $\beta$ is the maximum $g$ of all conditional probabilities, which satisfies the constraints $R\left(a_{N} \mid[o]\right) \leq$ $R\left(a_{P} \mid[o]\right)$ and $R\left(a_{N} \mid[o]\right) \leq R\left(a_{B} \mid[o]\right)$. Moreover, Liu proved that the equivalent model is mathematically equivalent to the decision-theoretic rough sets.

As the feasibility and validity of the idea have been verified in the literature, we apply the equivalent model in order to solve the evaluation problems of probabilistic linguistics and to obtain the probability threshold and three decision rules in Section 3.

\section{Description of Three-Way Decision Problems Based on Evaluation of Probabilistic Linguistic Term Sets}

The three-way decision problems based on evaluation of PLTSs is composed of two states $S=\{C, \neg C\} \triangleq\{P, N\}$ and three actions $A=\left\{a_{P}, a_{B}, a_{N}\right\}$. In addition, the loss function with different actions of decision-making in different states is given with PLTSs. Based on the heuristic knowledge, the value of loss function in multiple objects $O=$ $\left\{o_{1}, o_{2}, \ldots, o_{m}\right\}$ is given by experts with PLTSs, which is shown in Table 2. According to the related data in Table 2, how do we get the thresholds and rules of three-way decisions for each object $o_{i}(i=1,2, \ldots, m)$ ? For each object in Table 2, a proper case of a loss function is as follows: $L_{P P}^{i} \prec L_{B P}^{i} \prec L_{B P}^{i}, L_{N N}^{i} \prec L_{B N}^{i} \prec L_{P N}^{i}$.

Table 2. The loss function matrix of a Probabilistic Linguistic Term (PLT) with multiple objects.

\begin{tabular}{ccccccc}
\hline & $\lambda_{P P}$ & $\lambda_{P N}$ & $\lambda_{B P}$ & $\lambda_{B N}$ & $\lambda_{N P}$ & $\lambda_{N N}$ \\
\hline$o_{1}$ & $\lambda_{P P}^{1}=L_{P P}^{1}(P)$ & $\lambda_{P N}^{1}=L_{P N}^{1}(P)$ & $\lambda_{B P}^{1}=L_{B P}^{1}(P)$ & $\lambda_{B N}^{1}=L_{B N}^{1}(P)$ & $\lambda_{N P}^{1}=L_{N P}^{1}(P)$ & $\lambda_{N N}^{1}=L_{N N}^{1}(P)$ \\
\hline$o_{2}$ & $\lambda_{P P}^{2}=L_{P P}^{2}(P)$ & $\lambda_{P N}^{2}=L_{P N}^{2}(P)$ & $\lambda_{B P}^{2}=L_{B P}^{2}(P)$ & $\lambda_{B N}^{2}=L_{B N}^{2}(P)$ & $\lambda_{N P}^{2}=L_{N P}^{2}(P)$ & $\lambda_{N N}^{2}=L_{N N}^{2}(P)$ \\
\hline$\ldots$ & $\ldots$ & $\ldots$ & $\ldots$ & $\ldots$ & $\ldots$ & $\ldots$ \\
\hline$o_{i}$ & $\lambda_{P P}^{i}=L_{P P}^{i}(P)$ & $\lambda_{P N}^{i}=L_{P N}^{i}(P)$ & $\lambda_{B P}^{i}=L_{B P}^{i}(P)$ & $\lambda_{B N}^{i}=L_{B N}^{i}(P)$ & $\lambda_{N P}^{i}=L_{N P}^{i}(P)$ & $\lambda_{N N}^{i}=L_{N N}^{i}(P)$ \\
\hline$\ldots$ & $\ldots$ & $\ldots$ & $\ldots$ & $\ldots$ & $\ldots$ & $\ldots$ \\
\hline$o_{m}$ & $\lambda_{P P}^{m}=L_{P P}^{m}(P)$ & $\lambda_{P N}^{m}=L_{P N}^{m}(P)$ & $\lambda_{B P}^{m}=L_{B P}^{m}(P)$ & $\lambda_{B N}^{m}=L_{B N}^{m}(P)$ & $\lambda_{N P}^{m}=L_{N P}^{m}(P)$ & $\lambda_{N N}^{m}=L_{N N}^{m}(P)$ \\
\hline
\end{tabular}

A DTRS(s)model with probabilistic linguistic terms is constructed based on the loss function matrix in Table 2 and its key is the order of PLTSs. Aiming at the problem, the score and deviation degree of PLTSs were put forward. 
Definition 9. Ref. [32] Let $L(p)=\left\{L^{(k)}\left(p^{(k)}\right) \mid L^{(k)} \in S, k=1,2, \cdots, \# L(p)\right\}$ be a PLTS and $r_{k}$ be the subscript of linguistic term $L^{(k)}$. Then, the score of $L(p)$ is

$$
E(L(p))=s_{\bar{\alpha}}
$$

where $\bar{\alpha}=\frac{\sum_{k=1}^{\# L(p)} r^{(k)} p^{(k)}}{\sum_{k=1}^{\# L(p)} p^{(k)}}$.

Definition 10. Ref. [32] Let $L(p)=\left\{L^{(k)}\left(p^{(k)}\right) \mid L^{(k)} \in S, k=1,2, \cdots, \# L(p)\right\}$ be a PLTS and $r_{k}$ be the subscript of linguistic term $L^{(k)}$. Then, the deviation degree of $L(p)$ is

$$
\sigma(L(p))=\frac{\left(\sum_{k=1}^{\# L(p)}\left(p^{(k)}\left(r^{(k)}-\bar{\alpha}\right)\right)^{2}\right)^{\frac{1}{2}}}{\sum_{k=1}^{\# L(p)} p^{(k)}}
$$

Definition 11. Ref. [32] For PLTSs $L_{1}(p)$ and $L_{2}(p), E\left(L_{1}(p)\right)$ and $E\left(L_{2}(p)\right)$ are their scores, respectively, and $\sigma\left(L_{1}(p)\right)$ and $\sigma\left(L_{2}(p)\right)$ are their deviation degree, respectively. Then, we have the following rules:

(1) If $E\left(L_{1}(p)\right)>E\left(L_{2}(p)\right)$, then $L_{1}(p)>L_{2}(p)$;

(2) If $E\left(L_{1}(p)\right)<E\left(L_{2}(p)\right)$, then $L_{1}(p)<L_{2}(p)$;

(3) If $E\left(L_{1}(p)\right)=E\left(L_{2}(p)\right)$, then

(1) If $\sigma\left(L_{1}(p)\right)>\sigma\left(L_{2}(p)\right)$, then $L_{1}(p)>L_{2}(p)$;

(2) If $\sigma\left(L_{1}(p)\right)=\sigma\left(L_{2}(p)\right)$, then $L_{1}(p) \approx L_{2}(p)$;

(3) If $\sigma\left(L_{1}(p)\right)<\sigma\left(L_{2}(p)\right)$, then $L_{1}(p)<L_{2}(p)$.

According to Definition 11, the larger the score function is, the better the corresponding PLTS is, and when the score function is equal, the smaller the deviation function, the better the PLTS is. As $E\left(L_{1}(p)\right)=E\left(L_{2}(p)\right)$ is rare, we simplify the rules as follows:

$\left(1^{*}\right)$ If $E\left(L_{1}(p)\right)>E\left(L_{2}(p)\right)$, then $L_{1}(p)>L_{2}(p)$;

$\left(2^{*}\right)$ If $E\left(L_{1}(p)\right)<E\left(L_{2}(p)\right)$, then $L_{1}(p)<L_{2}(p)$;

$\left(3^{*}\right)$ If $E\left(L_{1}(p)\right)=E\left(L_{2}(p)\right)$, then $L_{1}(p) \approx L_{2}(p)$.

Therefore, to compare the size of two PLTSs, we just need to calculate and compare the values of their score function.

Based on the basic operations of PLTSs, the loss function of each object $o_{i}$ under the action $a_{P}, a_{B}$, and $a_{N}$ and can be recorded as $\widetilde{R}\left(a_{P} \mid\left[o_{i}\right]\right), \widetilde{R}\left(a_{B} \mid\left[o_{i}\right]\right)$, and $\widetilde{R}\left(a_{N} \mid\left[o_{i}\right]\right)$; then,

$$
\begin{aligned}
& \widetilde{R}\left(a_{\bullet} \mid\left[o_{i}\right]\right)=g_{i} \lambda_{\bullet P}^{i} \bigoplus h_{i} \lambda_{\bullet N}^{i}=g_{i} L_{\bullet P}^{i} \bigoplus h_{i} L_{\bullet N}^{i} \\
& =\cup_{L_{\bullet P}^{i(k)}\left(P^{(k)}\right) \in L_{\bullet P}(p), L_{\bullet N}^{i(k)}\left(P^{(k)}\right) \in L_{\bullet N}(p)}\left\{g_{i} L_{\bullet P}^{i(k)}\left(P^{(k)}\right) \bigoplus h_{i} L_{\bullet N}^{i(k)}\left(P^{(k)}\right)\right\} \\
& (\bullet=P, B, N)
\end{aligned}
$$

Obviously, the loss function $\widetilde{R}\left(a_{\bullet} \mid\left[o_{i}\right]\right)$ is still a PLTS(s). For the convenience of subsequent description, it is recorded as

$$
\begin{aligned}
& Q_{\bullet}^{i}(p)=\left\{Q_{\bullet}^{i(k)}\left(P^{(k)}\right) \mid Q_{\bullet}^{i(k)}\left(P^{(k)}=g_{i} L_{\bullet P}^{i(k)}\left(P^{(k)}\right) \bigoplus h_{i} L_{\bullet N}^{i(k)}\left(P^{(k)}\right)\right.\right. \\
& \left.L_{\bullet P}^{i(k)}\left(P^{(k)}\right) \in L_{\bullet P}(p), L_{\bullet N}^{i(k)}\left(P^{(k)}\right) \in L_{\bullet N}(p), k=1,2, \cdots, \# Q_{\bullet}^{i}(p)\right\}
\end{aligned}
$$

According to Bayesian decision rule for minimum risk, the following rules are obtained:

(P4) If $\widetilde{R}\left(a_{P} \mid\left[o_{i}\right]\right) \preceq \widetilde{R}\left(a_{B} \mid\left[o_{i}\right]\right)$ and $\widetilde{R}\left(a_{P} \mid\left[o_{i}\right]\right) \preceq \widetilde{R}\left(a_{N} \mid\left[o_{i}\right]\right)$, then $o \in \operatorname{POS}(C)$.

(B4) If $\widetilde{R}\left(a_{B} \mid\left[o_{i}\right]\right) \preceq \widetilde{R}\left(a_{P} \mid\left[o_{i}\right]\right)$ and $\widetilde{R}\left(a_{B} \mid\left[o_{i}\right]\right) \preceq \widetilde{R}\left(a_{N} \mid\left[o_{i}\right]\right)$, then $o \in B N D(C)$.

(N4) If $\widetilde{R}\left(a_{N} \mid\left[o_{i}\right]\right) \preceq \widetilde{R}\left(a_{P} \mid\left[o_{i}\right]\right)$ and $\widetilde{R}\left(a_{N} \mid\left[o_{i}\right]\right) \preceq \widetilde{R}\left(a_{B} \mid\left[o_{i}\right]\right)$, then $o \in N E G(C)$.

From the decision rule (P4)-(N4), the following proposition can be obtained through the ranking method of Definition 11. 
Proposition 1. Let $\widetilde{R}\left(a_{\bullet} \mid\left[o_{i}\right]\right)(\bullet=P, B, N)$ be a loss function of each object $o_{i}$ under the action $a_{\bullet} ;$ then, the score and deviation degree function is noted as

$$
E\left(\widetilde{R}\left(a_{\bullet} \mid\left[o_{i}\right]\right)\right)=E\left(Q_{\bullet}^{i}(p)\right)=s_{\bar{\alpha}}^{i}
$$

where $\bar{\alpha}=\frac{\sum_{k=1}^{\# Q_{0}^{i}(p)} r^{(k)} p^{(k)}}{\sum_{k=1}^{\left.\# Q_{0}^{i}(p)\right)} p^{(k)}}$.

For the above linear programming problems, we can use particle swarm optimization techniques that are presented in [40] to search their optimal solution and induce the rules of three-way decisions. We first construct the following linear reformulated model:

$$
\begin{aligned}
& \widetilde{\alpha}_{i}^{(o p t)}-\text { model : } \\
& \widetilde{\alpha}_{i}^{(o p t)}=\min g \\
& \text { s.t. }\left\{\begin{array}{l}
g_{i}+h_{i}=1, h_{i} \geq 0 \\
\frac{\sum_{k=1}^{\# Q_{P}^{i}(p)} r^{(k)} p^{(k)}}{\sum_{k=1}^{\left.\# Q_{P}^{i}(p)\right)} p^{(k)}} \leq \frac{\sum_{k=1}^{\# Q_{B}^{i}(p)} r^{(k)} p^{(k)}}{\sum_{k=1}^{\left.\# Q_{B}^{i}(p)\right)} p^{(k)}} \\
\frac{\sum_{k=1}^{\# Q_{p}^{(p)}} r^{(k)} p^{(k)}}{\sum_{k=1}^{\left.\# Q_{P}^{i}(p)\right)} p^{(k)}} \leq \frac{\sum_{k=1}^{\# Q_{B}^{i}(p)} r^{(k)} p^{(k)}}{\sum_{k=1}^{\left.\# Q_{B}^{i}(p)\right)} p^{(k)}}
\end{array}\right. \\
& \widetilde{\beta}_{i}^{(o p t)}-\text { model : } \\
& \widetilde{\beta}_{i}^{(o p t)}=\max g \\
& \text { s.t. }\left\{\begin{array}{l}
g_{i}+h_{i}=1, h_{i} \geq 0 \\
\frac{\sum_{k=1}^{\# Q_{N}^{i}(p)} r^{(k)} p^{(k)}}{\sum_{k=1}^{* Q_{N}^{i}(p)} p^{(k)}} \leq \frac{\sum_{k=1}^{\# Q_{p}^{i}(p)} r^{(k)} p^{(k)}}{\sum_{k=1}^{\left.\# Q_{p}^{i}(p)\right)} p^{(k)}} \\
\frac{\sum_{k=1}^{\# Q_{N}^{1}(p)} r^{(k)} p^{(k)}}{\sum_{k=1}^{\left.\# Q_{N}^{i}(p)\right)} p^{(k)}} \leq \frac{\sum_{k=1}^{\# Q_{N}^{(p)}} r^{(k)} p^{(k)}}{\sum_{k=1}^{\left.\# Q_{N}^{i}(p)\right)} p^{(k)}}
\end{array}\right.
\end{aligned}
$$

For linear programming problems, we can directly find the optimal solution through the Linprog function in MATLAB[41]. Next, we give the following theorems to ensure the existence and uniqueness of the optimal solution.

Theorem 1. The feasible region of $\widetilde{\alpha}_{i}^{(o p t)}$-model is $D \subseteq\left\{\left(g_{i}, h_{i}\right) \mid g_{i} \geq 0, h_{i} \leq 1, g_{i}+h_{i}=1\right\}$, and $(0,1) \in D$. Obviously, the feasible region is a nonempty closed bounded set. To deal with the linear programming problems, when the feasible region is a closed bounded region or an unclosedunbounded region, an optimal solution must be existed. Moreover, through the simplex method, the test number of all nonbasic variables in the optimal form is negative. Then, it can be determined that the linear programming problem has a unique optimal solution. Likewise, a unique optimal solution can also be obtained in $\widetilde{\beta}_{i}^{(\text {opt })}$-model.

\section{Method to Determining the Threshold Pair and Rules of 3WDs Based on the Evaluation of PLTSs}

To deal with the evaluation data in Table 2, we propose an optimization based on $\widetilde{\alpha}_{i}^{(o p t)}$-model and $\widetilde{\beta}_{i}^{(o p t)}-\operatorname{model}(i=1,2, \ldots, m)$, which can get the rules of three-way decisions directly.

Step 1: Constricting $\widetilde{\alpha}_{i}^{(o p t)}$-model and $\widetilde{\beta}_{i}^{(o p t)}-$ model through the loss function matrix with multiple objects in Table 2.

Step 2: This step involves calculating the optimal results of $\widetilde{\alpha}_{i}^{(o p t)}-$ model and $\widetilde{\beta}_{i}^{(o p t)}-$ model $(i=1,2, \ldots, m)$, and supposing the optimal results $\widetilde{\alpha}_{i}^{o p t}$ and $\widetilde{\beta}_{i}^{o p t}$. 
(i) If $\widetilde{\alpha}_{i}^{(o p t)}>\widetilde{\beta}_{i}^{(o p t)}$, then DMs adopt the three-way decision model.

(P6) If $\operatorname{Pr}(C \mid[o]) \geq \widetilde{\alpha}_{i}^{o p t}$, then $o_{i} \in \operatorname{POS}(C)$.

(B6) If $\widetilde{\beta}_{i}^{\text {opt }}<\operatorname{Pr}(C \mid[o])<\widetilde{\alpha}_{i}^{\text {opt }}$, then $o_{i} \in B N D(C)$.

(N6) If $\operatorname{Pr}(C \mid[o]) \leq \widetilde{\beta}_{i}^{o p t}$, then $o_{i} \in \operatorname{NEG}(C)$.

(ii) If $\widetilde{\alpha}_{i}^{(o p t)}=\widetilde{\beta}_{i}^{(o p t)}$, then the DMs adopt a two-way decision model.

(P7) If $\operatorname{Pr}(C \mid[o]) \geq \widetilde{\alpha}_{i}^{o p t}$, then $o_{i} \in \operatorname{POS}(C)$.

(N7) If $\operatorname{Pr}(C \mid[o])<\widetilde{\alpha}_{i}^{o p t}$, then $o_{i} \in N E G(C)$.

Based on the trisecting-and-acting model in Figure 1 and the above steps, a three-way decision model based on PLTS(s) evaluations is proposed in Figure 2.

Based on the above model, the specific algorithm is given below(Algorithm 1).

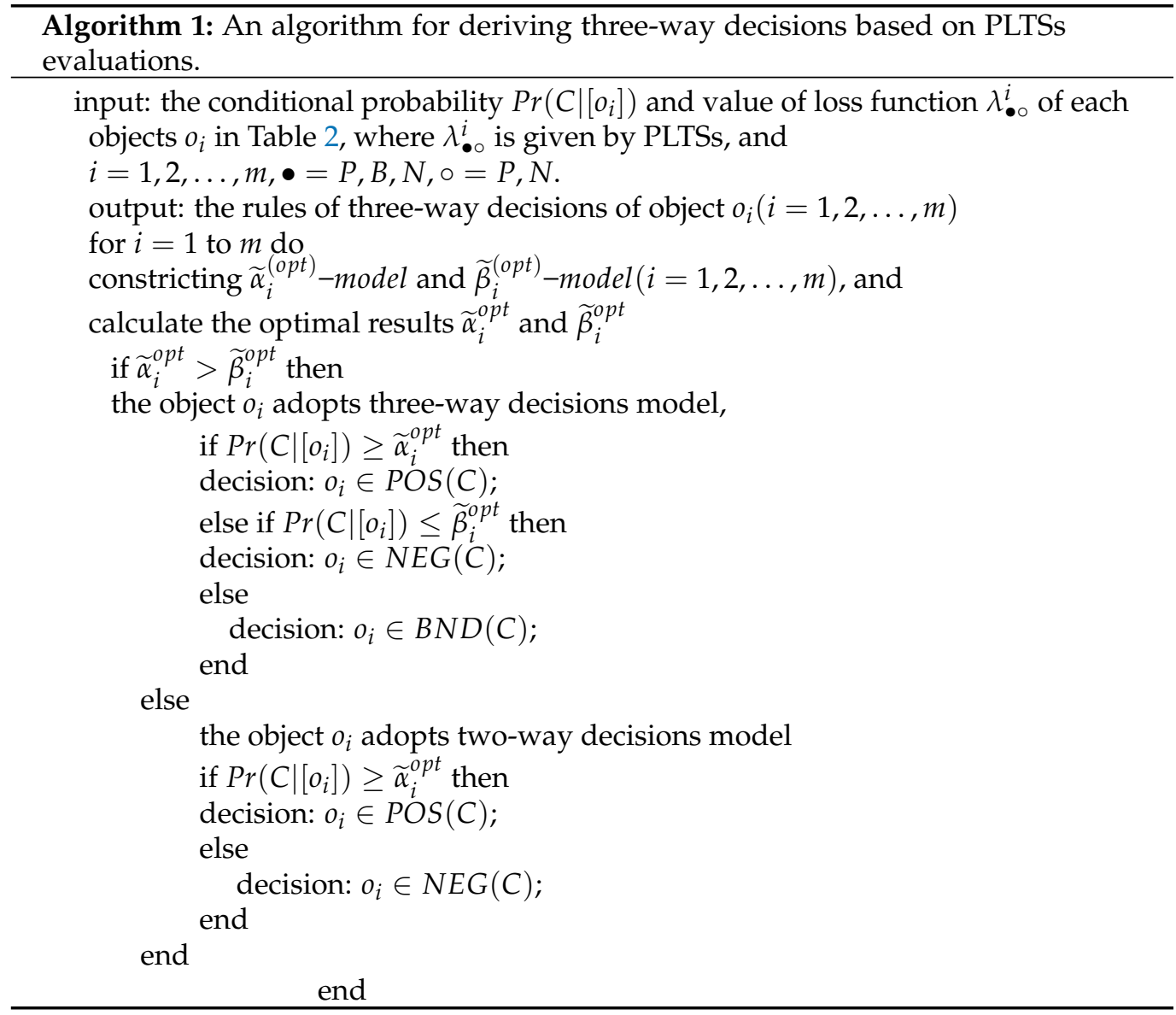




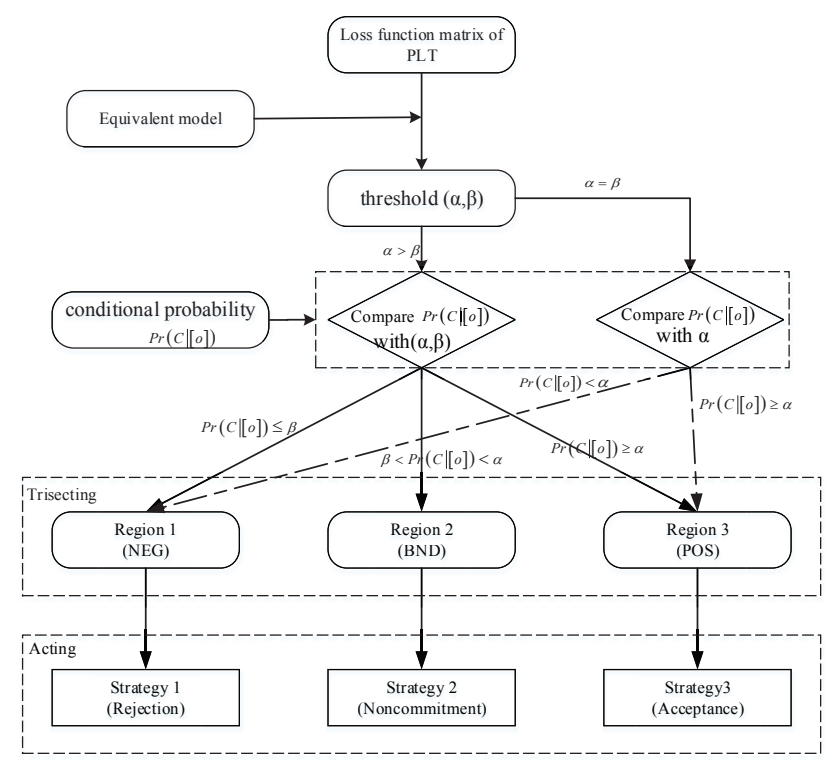

Figure 2. A three-way decision model based on PLTS(s) evaluations.

\section{Example Analysis and Comparison Discussion}

\subsection{Example Analysis}

In order to verify the validity of the proposed method, consider three bidding companies that can be divided into good companies and bad companies, depending on the previous experience of project contracting. These parties participate in project bidding simultaneously and the tendering company may take any of the three actions (accept, further consider, or abandon) against the bidding companies. However, the loss function for different types of bidding companies with three different actions is diverse. For example, it may lead to tenderergreat economic benefits when choosing to accept a good bidding company. Conversely, it may lead to huge economic losses and time wastage. Therefore, in order to make reasonable scientific decisions in the bidding process, the tendering company hires the relevant experts to conduct a comprehensive evaluation of the bidding project at the beginning period of preparation. According to the prior knowledge, the loss function, which is outlined in Table 3 [33], is given by an expert in the field in the form of probability linguistic terms. Meanwhile, to simplify the problem, the conditional probabilities are assumed as $\operatorname{Pr}\left(C \mid\left[o_{1}\right]\right)=0.7035, \operatorname{Pr}\left(C \mid\left[o_{2}\right]\right)=0.6535, \operatorname{Pr}\left(C \mid\left[o_{3}\right]\right)=0.3545$ in advance. In the case where the above information is known, which of the three bidding companies should the tendering company choose?

Step 1: Taking company $o_{1}$ as an example, $\widetilde{\alpha}_{1}^{(o p t)}-$ model and $\widetilde{\beta}_{1}^{(o p t)}-$ model are constructed based on the equivalent model in Section 2 and the loss function in Table 3, and so do the other companies.

Table 3. The loss function matrix of PLT with multiple objects.

\begin{tabular}{cccc}
\hline & $\boldsymbol{o}_{\mathbf{1}}$ & $\boldsymbol{o}_{\mathbf{2}}$ & $\boldsymbol{o}_{\mathbf{3}}$ \\
\hline$\lambda_{P P}$ & $\left\{s_{1}(0.40), s_{2}(0.45), s_{3}(0.15)\right\}$ & $\left\{s_{1}(0.80), s_{2}(0.10), s_{3}(0.10)\right\}$ & $\left\{s_{1}(0.15), s_{2}(0.25), s_{3}(0.60)\right\}$ \\
\hline$\lambda_{P N}$ & $\left\{s_{1}(0.25), s_{2}(0.25), s_{3}(0.50)\right\}$ & $\left\{s_{1}(0.05), s_{2}(0.20), s_{3}(0.75)\right\}$ & $\left\{s_{1}(0.70), s_{2}(0.15), s_{3}(0.15)\right\}$ \\
\hline$\lambda_{B P}$ & $\left\{s_{1}(0.30), s_{2}(0.55), s_{3}(0.15)\right\}$ & $\left\{s_{1}(0.70), s_{2}(0.10), s_{3}(0.15)\right\}$ & $\left\{s_{1}(0.15), s_{2}(0.20), s_{3}(0.65)\right\}$ \\
\hline$\lambda_{B N}$ & $\left\{s_{1}(0.25), s_{2}(0.35), s_{3}(0.40)\right\}$ & $\left\{s_{1}(0.10), s_{2}(0.25), s_{3}(0.65)\right\}$ & $\left\{s_{1}(0.75), s_{2}(0.15), s_{3}(0.10)\right\}$ \\
\hline$\lambda_{N P}$ & $\left\{s_{1}(0.25), s_{2}(0.50), s_{3}(0.25)\right\}$ & $\left\{s_{1}(0.65), s_{2}(0.10), s_{3}(0.25)\right\}$ & $\left\{s_{1}(0.15), s_{2}(0.15), s_{3}(0.70)\right\}$ \\
\hline$\lambda_{N N}$ & $\left\{s_{1}(0.35), s_{2}(0.25), s_{3}(0.40)\right\}$ & $\left\{s_{1}(0.10), s_{2}(0.35), s_{3}(0.55)\right\}$ & $\left\{s_{1}(0.80), s_{2}(0.15), s_{3}(0.05)\right\}$ \\
\hline
\end{tabular}

The expected losses for $o_{1}$ with three different actions based on the data in Table 3 and Formula (13): 


$$
\begin{aligned}
& Q_{P}^{1}(p)=\left\{s_{1}\left(0.40 g_{1}+0.25 h_{1}\right), s_{2}\left(0.45 g_{1}+0.25 h_{1}\right), s_{3}\left(0.15 g_{1}+0.50 h_{1}\right)\right\} \\
& Q_{B}^{1}(p)=\left\{s_{1}\left(0.30 g_{1}+0.25 h_{1}\right), s_{2}\left(0.55 g_{1}+0.35 h_{1}\right), s_{3}\left(0.15 g_{1}+0.40 h_{1}\right)\right\} \\
& Q_{N}^{1}(p)=\left\{s_{1}\left(0.25 g_{1}+0.35 h_{1}\right), s_{2}\left(0.50 g_{1}+0.25 h_{1}\right), s_{3}\left(0.25 g_{1}+0.40 h_{1}\right)\right\}
\end{aligned}
$$

$$
\begin{aligned}
& \widetilde{\alpha}_{1}^{(o p t)}-\text { model : } \\
& \widetilde{\alpha}_{1}^{(o p t)}=\min g_{1} \\
& \text { s.t. }\left\{\begin{array}{l}
g_{1}+h_{1}=1, h_{1} \geq 0 \\
\frac{0.4 g_{1}+0.25 h_{1}+\left(0.45 g_{1}+0.25 h_{1}\right) \times 2+\left(0.15 g_{1}+0.50 h_{1}\right) \times 3}{0.3 g_{1}+0.25 h_{1}+0.45 g_{1}+0.25 h_{1}+0.15 g_{1}+0.50 h_{1}} \leq \frac{0.3 g_{1}+0.25 h_{1}+\left(0.55 g_{1}+0.35 h_{1}\right) \times 2+\left(0.15 g_{1}+0.4 h_{1}\right) \times 3}{0.3 g_{1}+0.25 h_{1}+0.55 g_{1}+0.35 h_{1}+0.15 g_{1}+0.40 h_{1}} \\
\frac{0.4 g_{1}+0.25 h_{1}+\left(0.45 g_{1}+0.25 h_{1}\right) \times 2+\left(0.15 g_{1}+0.50 h_{1}\right) \times 3}{0.3 g_{1}+0.25 h_{1}+0.45 g_{1}+0.25 h_{1}+0.15 g_{1}+0.50 h_{1}} \leq \frac{0.25 g_{1}+0.35 h_{1}+\left(0.5 g_{1}+0.25 h_{1}\right) \times 2+\left(0.25 g_{1}+0.4 h_{1}\right) \times 3}{0.25 g_{1}+0.35 h_{1}+0.5 g_{1}+0.25 h_{1}+0.25 g_{1}+0.4 h_{1}}
\end{array}\right. \\
& \widetilde{\beta}_{1}^{(o p t)}-\text { model : } \\
& \widetilde{\beta}_{1}^{(o p t)}=\max g_{1} \\
& \text { s.t. }\left\{\begin{array}{l}
g_{1}+h_{1}=1, h_{1} \geq 0 \\
\frac{0.25 g_{1}+0.35 h_{1}+\left(0.5 g_{1}+0.25 h_{1}\right) \times 2+\left(0.25 g_{1}+0.4 h_{1}\right) \times 3}{0.25 g_{1}+0.35 h_{1}+0.5 g_{1}+0.25 h_{1}+0.25 g_{1}+0.4 h_{1}} \leq \frac{0.4 g_{1}+0.25 h_{1}+\left(0.45 g_{1}+0.25 h_{1}\right) \times 2+\left(0.15 g_{1}+0.50 h_{1}\right) \times 3}{0.3 g_{1}+0.25 h_{1}+0.45 g_{1}+0.25 h_{1}+0.15 g_{1}+0.50 h_{1}} \\
\frac{0.25 g_{1}+0.35 h_{1}+\left(0.5 g_{1}+0.25 h_{1}\right) \times 2+\left(0.25 g_{1}+0.4 h_{1}\right) \times 3}{0.25 g_{1}+0.35 h_{1}+0.5 g_{1}+0.25 h_{1}+0.25 g_{1}+0.4 h_{1}} \leq \frac{0.3 g_{1}+0.25 h_{1}+\left(0.55 g_{1}+0.35 h_{1}\right) \times 2+\left(0.15 g_{1}+0.4 h_{1}\right) \times 3}{0.3 g_{1}+0.25 h_{1}+0.55 g_{1}+0.35 h_{1}+0.15 g_{1}+0.40 h_{1}}
\end{array}\right.
\end{aligned}
$$

Step 2: By calculating the optimal solutions in $\widetilde{\alpha}_{1}^{(o p t)}-$ model and $\widetilde{\beta}_{1}^{(o p t)}-$ model the optimal solution and three-way decision rules are derived and given in Table 4.

Table 4 shows that the probability thresholds of three-way decisions about three bidding companies are $\widetilde{\alpha}_{1}^{(o p t)}=0.5, \widetilde{\beta}_{1}^{(o p t)}=0.4, \widetilde{\alpha}_{2}^{(o p t)}=0.75, \widetilde{\beta}_{2}^{(o p t)}=0.2857$, and $\widetilde{\alpha}_{3}^{(o p t)}=\widetilde{\beta}_{3}^{(o p t)}=0.6667$, respectively. Consequently, the company should accept $o_{1}$ and reject $o_{3}$ under the condition probability given in Table 4 , and $o_{2}$ needs further consideration.

Table 4. The probability thresholds and three-way decisions of three companies using the proposed method.

\begin{tabular}{cccc}
\hline $\boldsymbol{O}$ & $\boldsymbol{o}_{\mathbf{1}}$ & $\boldsymbol{o}_{\mathbf{2}}$ & $\boldsymbol{o}_{\mathbf{3}}$ \\
\hline $\operatorname{Pr}\left(C \mid\left[o_{i}\right]\right)$ & 0.7035 & 0.6535 & 0.3545 \\
\hline$\widetilde{\alpha}_{i}^{\text {opt }}$ & 0.5 & 0.75 & 0.6667 \\
\hline$\widetilde{\beta}_{i}^{\text {opt }}$ & 0.4 & 0.2857 & 0.6667 \\
\hline Decision mode & three-way decisions & three-way decisions & two-way decisions \\
\hline Decision rules & POS $(\mathrm{C})$ & $\mathrm{BND}(\mathrm{C})$ & $\mathrm{NEG}(\mathrm{C})$ \\
\hline
\end{tabular}

From the analysis results, the method proposed in this paper can be applied to deal with the problem that the loss function is in the form of hesitation fuzzy linguistic terms and probability linguistic terms. We could determine the probability threshold of the three decisions and obtain the relevant decision rules simultaneously.

\subsection{Comparison Discussion}

Approaches based on the classical DTRSs are here to analyze the example in [2]. According to Formula (13), we calculate the $\widetilde{R}\left(a_{\bullet} \mid\left[o_{i}\right]\right)(\bullet=P, B, N)$ of the three companies. By the use of Formula (14), the threshold pairs are obtained. Then, we found that the threshold pairs are the same as those generated by our method.

Compared to the approaches, our method has the following advantages. 
(1) Our method can determine the thresholds stated herein and overcome the drawback of not capturing these thresholds in various scenarios.

(2) Our method can be used for the determination of the thresholds with the loss function, which are evaluated by other semantics. Therefore, our approach is more universal than others.

\section{Conclusions}

According to the existing method in some practical situations, evaluating concrete data and determining the probability threshold of the three-way decisions are difficult. This paper studies an optimal model based on the loss function evaluated by PLTSs. Quoting the equivalent model of DTRSs, the optimization models with a loss function of the probabilistic linguistic terms are used to determine the three-way decision threshold. The analysis results of the following examples show that the method proposed in this paper can effectively solve problems that are difficult to determine, such as the probability thresholds of three-way decisions evaluated by fuzzy language. As an extension of the method in this work, we propose that our method can be applied to determine the application of fuzzy set theory in the actual decision environment; it also provides a new idea for the follow-up study of fuzzy language evaluation.

In order to further explore the influence of different ranking methods on decisionmaking results, we hope to introduce the concepts of both the fuzzy entropy and hesitant entropy of PLTSs in three-way decisions.

Author Contributions: Conceptualization and methodology, P.G. and J.L.; formal analysis and writing - original draft preparation, P.G.; writing—review, J.L.; supervision and funding acquisition, X.Z. All authors have read and agreed to the published version of the manuscript.

Funding: This research was funded by the National Nature Science Foundation of China (NO.61876079), the Foundation for Young Talents in Higher Education of Guangdong Province (NO.2019WQNCXO27), the Foundation of the 13th Five-Year Plan for Young Talents in Philosophy and Social Science of Guangdong Province (NO.GD20YGL13), the Start-up Research Science Found of Shantou University (NO.STF19025), and GuangDong Basic and Applied Basic Research Foundation (NO.2020A1515110434).

Institutional Review Board Statement: Not applicable.

Informed Consent Statement: Not applicable.

Data Availability Statement: Not applicable.

Conflicts of Interest: The authors declare no conflicts of interest.

\begin{tabular}{|c|c|}
\hline \multicolumn{2}{|c|}{ Abbreviations } \\
\hline \multicolumn{2}{|c|}{ The following abbreviations are used in this manuscript: } \\
\hline 3WDs & three-way decisions \\
\hline PLTSs & Probabilistic Linguistic Terms Sets \\
\hline DTRSs & Decision-theoretic rough sets \\
\hline DMs & decision-makers \\
\hline INs & interval numbers \\
\hline TFs & triangle fuzzy numbers \\
\hline IFSs & Intuitionistic Fuzzy Sets \\
\hline LTSs & Linguistic Term Sets \\
\hline HFSs & Hesitant Fuzzy Sets \\
\hline HFLSs & Hesitant Fuzzy Linguistic Term Sets \\
\hline IVPLTS & Interval-valued Probabilistic Linguistic Term sets \\
\hline
\end{tabular}

\section{References}

1. Yao, Y.Y.; Wong, S.; Lingras, P. A decision-theoretical rough set model. Methodol. Intell. Syst. 1990, 5, 17-24.

2. Yao, Y.Y. Three-way decisions with probabilistic rough sets. Inf. Sci. 2010, 180, 341-353. [CrossRef] 
3. Yao, Y.Y. Three-way granular computing, rough sets, and formal concept analysis. Int. J. Approx. Reason. 2020, 116, $285-306$. [CrossRef]

4. Hu, B.Q.; Wong, H.; Yiu, K. On two novel types of three-way decisions in three-way decision spaces. Int. J. Approx. Reason. 2017, 82, 285-306. [CrossRef]

5. Li, X.N. Three-way fuzzy matroids and granular computing. Int. J. Approx. Reason. 2019, 114, 44-50. [CrossRef]

6. Liu, D.; Liang, D.C.; Wang, C.C. A novel three-way decision model based on incomplete information system. Knowl.-Based Syst. 2016, 91, 32-45. [CrossRef]

7. Yang, X.; Li, T.R.; Liu, D.; Fujita, H. A temporal-spatial composite sequential approach of three-way granular computing. Inf. Sci. 2019, 486, 171-189. [CrossRef]

8. Fang, Y.; Min, F. Cost-sensitive approximate attribute reduction with three-way decisions. Int. J. Approx. Reason. 2019, 104, 148-165. [CrossRef]

9. Zhang, X.Y.; Miao, D.Q. Three-way attribute reducts. Int. J. Approx. Reason. 2017, 88, 401-434. [CrossRef]

10. Zhang, X.Y.; Yang, J.L.; Tang, L.Y. Three-way class-specific attribute reducts from the information viewpoint. Inf. Sci. 2020, 507, 840-872. [CrossRef]

11. Editor Peer Review: Its Strengths and Weakness. Available online: http:/ /hdl.handle.net/10822/941128 (accessed on 10 January 2021).

12. Zhang, H.R.; Min, F.; Tang, B.S. Regression-based three-way recommendation. Inf. Sci. 2017, 378, 444-461. [CrossRef]

13. Zhang, H.R.; Min, F. Three-way recommender systems based on random forests. Knowl.-Based Syst. 2016, 91, 275-286. [CrossRef]

14. Zhang, X.; Miao, D. Three-layer granular structures and three-way informational measures of a decision table. Inf. Sci. 2017, 412-413, 67-86. [CrossRef]

15. Sun, B.Z.; Ma, W.M.; Li, B.J.; Li, X.N. Three-way decisions approach to multiple attribute group decision making with linguistic information-based decision-theoretic rough fuzzy set. Int. J. Approx. Reason. 2018, 93, 424-442. [CrossRef]

16. Afridi, M.K.; Azam, N.; Yao, J.T.; Alanazi, E. A three-way clustering approach for handling missing data using GTRS. Int. J. Approx. Reason. 2018, 98, 11-24. [CrossRef]

17. Wang, P.X.; Yao, Y.Y. CE3: A three-way clustering method based on mathematical morphology. Knowl.-Based Syst. 2018, 155, 54-65. [CrossRef]

18. Li, J.H.; Huang, C.C.; Qi, J.J.; Qian, Y.H.; Liu, W.Q. Three-way cognitive concept learning via multi-granularity. Inf. Sci. 2017, 378, 244-263. [CrossRef]

19. Sakai, H.; Nakata, M.; Watada, J. NIS-Apriori-based rule generation with three-way decisions and its application system in SQL. Inf. Sci. 2020, 507, 755-771. [CrossRef]

20. Hu, J.H.; Chen, D.; Liang, P. A Novel Interval Three-Way Concept Lattice Model with Its Application in Medical Diagnosis. Mathematics 2019, 7, 103. [CrossRef]

21. Hu, J.H.; Chen, D.; Liang, P. Sequential three-way decision and granulation for cost-sensitive face recognition. Knowl.-Based Syst. 2015, 91, 241-251.

22. Li, H.X.; Zhou, X.Z. Risk Decision Making Based on Decision-theoretic Rough Set: A Three-way View Decision Model. Int. J. Comput. Intell. Syst. 2011, 4, 1-11. [CrossRef]

23. Liang, D.C.; Liu, D. Deriving three-way decisions from intuitionistic fuzzy decision-theoretic rough sets. Inf. Sci. 2015, 300, 28-48. [CrossRef]

24. Liang, D.C.; Liu, D. Systematic studies on three-way decisions with interval-valued decision-theoretic rough sets. Inf. Sci. 2014, 276, 186-203. [CrossRef]

25. Jia, X.Y.; Tang, Z.M.; Liao, W.H.; Shang, L. On an optimization representation of decision-theoretic rough set model. Int. J. Approx. Reason. 2014, 55, 156-166. [CrossRef]

26. Azam, N.; Zhang, Y.; Yao, J.T. Evaluation functions and decision conditions of three-way decisions with game-theoretic rough sets. Eur. J. Oper. Res. 2017, 261, 704-741. [CrossRef]

27. Liu, J.B.; Zhou, X.Z.; Huang, B.; Li, H.X.; Ju, H.R. Combining similarity and divergence measure for intuitionistic fuzzy information clustering. J. Intell. Fuzzy Syst. 2019, 36, 3195-3209. [CrossRef]

28. Liu, J.B.; Gu, P.P.; Zhou, X.Z.; Li, H.X. A method for intuitionistic fuzzy three-way group decisions based on optimization models. J. Nanjing Univ. (Nat. Sci.) 2018, 54, 944-956.

29. Zadeh, L.A. The concept of a linguistic variable and its application to approximate reasoning-II. Inf. Sci. 1975, 8, 301-357. [CrossRef]

30. Torra, V. Hesitant fuzzy sets. Int. J. Intell. Syst. 2010, 6, 529-539. [CrossRef]

31. Rodriguez, R.M.; Martinez, L.; Herrera, F. Hesitant fuzzy linguistic term sets for decision making. IEEE Trans. Fuzzy Syst. 2012, 20, 109-119. [CrossRef]

32. Pang, Q.; Wang, H.; Xu, Z. Probabilistic linguistic term dets in multi-attribute group decision making. Inf. Sci. 2016, 369, 128-143. [CrossRef]

33. Bai, C.; Zhang, R.; Shen, S.; Xin, F. Interval-valued probabilistic linguistic term sets in multi-criteria group decision making. Int. J. Intell. Syst. 2018, 33, 1301-1321. [CrossRef]

34. Delgado, M.; Verdegay, J.L.; Vila, M.A. Linguistic decision-making models. Int. J. Intell. Syst. 1992, 7, 479-492. [CrossRef] 
35. Rodriguez, R.M.; Martinez, L.; Herrera, F. A group decision making model dealing with comparative linguistic expressions based on hesitant fuzzy linguistic term sets. Inf. Sci. 2013, 241, 28-42. [CrossRef]

36. Zhu, B.; Herrera, Z.S.X. Consistency measures for hesitant fuzzy linguistic preference relations. IEEE Trans. Fuzzy Syst. 2014, 22, 35-45. [CrossRef]

37. Sang, Y.L.; Liang, J.Y.; Qian, Y.H. Decision-theoretic rough sets under dynamic granulation. Knowl.-Based Syst. 2016, 91, 84-92. [CrossRef]

38. Yao, Y.Y. Three-way decision and granular computing. Int. J. Approx. Reason. 2018, 103, 107-123. [CrossRef]

39. Liu, J.B.; Li, H.X.; Zhou, X.Z.; Huang, B.; Wang, T.X. An optimization-based formulation for three-way decision. Inf. Sci. 2019, 495, 185-214. [CrossRef]

40. Liu, J.B.; Li, H.X.; Huang, B.; Zhou, X.Z.; Zhang, L.B. Similarity-divergence intuitionistic fuzzy decision using particle swarm optimization. Appl. Soft Comput. 2019, 81, 105479. [CrossRef]

41. Yang, J. Application of MATLAB in Mechanical Optimal Design. Appl. Mech. Mater. 2014, 509, 111-117. [CrossRef] 